\title{
REMARKS ON THE PURE CRITICAL EXPONENT PROBLEM
}

\author{
E.N. DANCER
}

In this paper, we use geometric and analytic methods to study the existence of positive solutions of the pure critical exponent problem with Dirichlet boundary conditions. In particular we prove that there is no solution for domains which are nearly star-shaped and we show that being conformal to a star-shaped domain does not characterise the domains for which the problem has no solution. We also answer some questions of Rodriguez et al.

In this paper, we discuss the existence of positive solutions of the pure critical exponent problem

$$
\begin{array}{rll}
-\Delta u=u^{p} & \text { in } & \Omega \\
u=0 & \text { on } & \partial \Omega .
\end{array}
$$

Here $\Omega$ is a bounded domain in $R^{m}$ with smooth boundary, $m>2$ and $p$ is the critical exponent $(m+2)(m-2)^{-1}$. We discuss a slight generalisation of starshapedness namely weakly star shaped and then prove that, if $\Omega$ is $C^{2}$ close to a weakly star-shaped domain, then (1) has no positive solution. (We do not know if this is true for sign changing solutions.) In a companion paper [4], Kewei Zhang and I have proved a similar result for $p>(m+2)(m-2)^{-1}$ (and related results). The proofs there are quite different. As a consequence of my result above, it follows that (1) has no positive solution on $\Omega$ if $\Omega$ is $C^{2}$ close to a domain $D$ where $D$ is conformally equivalent to a bounded weakly star shaped domain. We then use this to show that there exists domains $\Omega$ which are not conformally equivalent to bounded weakly star shaped domain but (1) has no positive solution. This disposes of a natural conjecture for when (1) has a positive solution. In the process, we obtain a useful characterisation of which domains are conformal to bounded weakly star shaped domains. We use this to give a much simpler proof of a much stronger version of a result of Rodriguez, Comte and Lewandowski [12] and answer two questions there.

Received 23rd March, 1998

Copyright Clearance Centre, Inc. Serial-fee code: 0004-9729/98 \$A2.00+0.00. 


\section{WEAKLY STAR SHAPED DOMAINS}

A domain $\Omega \subseteq R^{n}$ is weakly star shaped if there exists $x_{0} \in \bar{\Omega}$ such that every half line through $x_{0}$ intersects $\bar{\Omega}$ in a connected set (possibly consisting only of $\left\{x_{0}\right\}$ ). This connected set is an "interval". This is basically the usual definition of star-shapedness except that we allow $x_{0}$ to belong to $\partial \Omega$. This is more convenient later. We refer to $x_{0}$ as a centre of $\Omega$. The following result is possibly known but we do not know a reference.

Proposition. Assume that $\Omega$ is a bounded $C^{2}$ domain. Then $\Omega$ is weakly star shaped with centre $x_{0}$ if and only if $\left\langle x-x_{0}, n(x)\right\rangle \geqslant 0$ on $\partial \Omega$ where $n(x)$ is the outward normal.

REMARK. Clearly, if $x_{0} \in \partial \Omega$, it suffices to assume the condition for $x$ not equal to $x_{0}$.

Proof: Since our conditions only involve the line segment joining $x_{0}$ to $x$ and the component of the normal in the direction of $x-x_{0}$, it suffices to assume $\Omega$ is 2-dimensional.

The condition is clearly necessary because if $\left\langle x-x_{0}, n(x)\right\rangle<0$, the outward normal at $x$ makes an obtuse angle with $x-x_{0}$ (in the direction away from $x_{0}$ ) and hence the outward normal makes an acute angle with $x_{0}-x$. Thus $\alpha x+(1-\alpha) x_{0} \notin \bar{\Omega}$ if $\alpha$ is close to 1 and less than 1 . (Since $\Omega$ is smooth, we must move out of $\Omega$ if we move in any direction making an acute angle with the outward normal). This contradicts the connectedness of $\bar{\Omega} \cap\left\{t x+(1-t) x_{0}: t \geqslant 0\right\}$.

To prove the condition is sufficient, assume $x_{1} \in \bar{\Omega}$ but $t_{0} x_{1}+\left(1-t_{0}\right) x_{0} \notin \bar{\Omega}$ for some $t_{0} \in(0,1)$. By approximating $x_{1}$ by points of int $\Omega$ and by a limit argument, we see that we can assume $x_{1} \in$ int $\Omega$. By a similar argument, the same condition holds for all points near $x_{1}$. We shall prove below that there exist points $\widehat{x}$ arbitrarily close to $x_{1}$ such that $\left\langle n\left(t \widehat{x}+(1-t) x_{0}\right),\left(\widehat{x}-x_{0}\right)\right\rangle \neq 0$ if $t \neq 1$ and $t \widehat{x}+(1-t) x_{0} \in \partial \Omega$. Assuming this for the moment, it follows that we can also assume that $x_{1}$ has this property. If $s x_{1}+(1-s) x_{0} \notin \bar{\Omega}$ where $s \in(0,1)$, let $\alpha=\inf \left\{t: t>0, \alpha x_{1}+(1-\alpha) x_{0} \in \bar{\Omega}\right\}$. Then $\alpha x_{1}+(1-\alpha) x_{0} \in \partial \Omega$ while $(\alpha-\varepsilon) x_{1}+(1-(\alpha-\varepsilon)) x_{0} \in \bar{\Omega}$ if $\varepsilon$ is small and positive. We then argue much as in the first part to check that $\left\langle n\left(\alpha x_{1}+(1-\alpha) x_{0}\right),\left(x_{1}-x_{0}\right)\right\rangle \geqslant$ 0 . But by our choice of $x_{1},\left\langle n\left(\alpha x_{1}+(1-\alpha) x_{0}\right),\left(x_{1}-x_{0}\right)\right\rangle \neq 0$. Hence $n\left(\alpha x_{1}+\right.$ $\left.(1-\alpha) x_{0}\right) \cdot\left(x_{1}-x_{0}\right)<0$ which contradicts our assumptions. This proves our claim except we have to prove that we can choose $x_{1}$ suitably.

In fact, we prove our claim that, for most $x_{1},\left\langle n\left(t x_{1}+(1-t) x_{0}\right),\left(x_{1}-x_{0}\right)\right\rangle \neq 0$ whenever $t x_{1}+(1-t) x_{0} \in \partial \Omega$. To see this we look at the $C^{1}$ map $S(x)=$ $\left\|x-x_{0}\right\|^{-1}\left(x-x_{0}\right)$ of $\partial \Omega \backslash\left\{x_{0}\right\}$ to $S^{1}$. By Sard's theorem, most values of $S$ in $S^{1}$ are regular values and hence it suffices to prove that if $x_{2} \in \partial \Omega \backslash\left\{x_{0}\right\}$ and $S^{\prime}\left(x_{2}\right)$ has zero 
kernel, then $\left\langle n\left(x_{2}\right),\left(x_{2}-x_{0}\right)\right\rangle \neq 0$. To see this, note that, if $\left\langle n\left(x_{2}\right),\left(x_{2}-x_{0}\right)\right\rangle=0$, then the tangent space to $\partial \Omega$ at $x_{2}$ is parallel to $x_{2}-x_{0}$ and hence the $C^{1}$ surface $\partial \Omega$ near $x_{2}$ is of the form $x_{2}+\widetilde{\alpha}\left(x_{2}-x_{0}\right)+o(\widetilde{\alpha})$ where $\widetilde{\alpha}$ is small. A simple computation then shows $S^{\prime}\left(x_{2}\right)\left(x_{2}-x_{0}\right)=0$ which proves our claim. This completes the proof.

REMARKS.

1. It is easy to construct examples of domains which are weakly star shaped but not star shaped. The advantage of weakly star shaped is that it follows easily from the proposition that if $\Omega_{n}$ is weakly star shaped and $\Omega_{n} \longrightarrow \Omega_{0}$ in the $C^{1}$ sense, then $\Omega_{0}$ is also weakly star shaped. (Thus the set of weakly star shaped domains is closed in a suitable topology.)

2. If $\Omega$ is weakly star shaped, $\bar{\Omega}$ is contractible and hence by duality theorems for manifolds (as in [7]), it follows that $\partial \Omega$ is connected.

3. If $\Omega$ is weakly star shaped and $x$ is the point of $\partial \Omega$ furthest from $x_{0}$, then $n(x)$ is parallel to $x-x_{0}$ and hence $\left\langle n(x),\left(x-x_{0}\right)\right\rangle>0$. Thus strict inequality must hold at some points of $\partial \Omega$.

4. It is easy to see that, if $\Omega$ is weakly star shaped and $x \in \Omega$, then $t x_{0}+(1-t) x \in$ $\Omega$ for $0<t<1$. (This uses that int $\bar{\Omega}=\Omega$ ) and this is in fact an equivalent condition. (The equivalence follows by taking closures.)

To complete this section, we discuss briefly which bounded domains are conformally equivalent to bounded weakly star shaped domains. We shall assume our domains are $C^{1}$, our conformal transformations are $C^{1}$ and $m \geqslant 3$. By Vaisala [13], any conformal transformation $C$ on an open set in $R^{n}$ can be extended to a conformal transformation on $R^{n}$. Note that conformal transformations which do not involve inversion are of no interest to use because these are compositions of orthogonal transformations, translations and the maps $x \longrightarrow r x$ where $r>0$ and hence preserve weak star-shapedness. A conformal transformation involving inversions can be uniquely written in the form $x \longrightarrow b+r\|x+a\|^{-2} \widetilde{T}(x+a)$ where $\widetilde{T}$ is an orthogonal transformation (see [13]). We refer to $b$ as the point at $\infty$. (It is the image of $\infty$ under the transformation.) Hence if $\Omega_{1}$ is bounded and weakly star-shaped and $T\left(\Omega_{1}\right)$ is bounded where $T$ is conformal and involves an inversion then $-a \notin \bar{\Omega}$, and if $x_{0}$ is a centre of $\bar{\Omega}_{1}$ then $T$ maps half lines segments from $x_{0}$ to infinity to planar circular semi arcs joining $T\left(x_{0}\right)$ to $b$. Moreover, by connectedness, $T\left(\bar{\Omega}_{1}\right)$ intersected with the same semi arc will be connected. Note that a semi arc is one of the two arcs joining $\bar{x}_{0}$ and $b$ on the circle. Since the argument is reversible we see that a bounded $C^{1}$ domain $\Omega$ is conformal to a weakly star shaped bounded domain if and only if there exists an $\bar{x}$ in $\bar{\Omega}$ and a $b \in R^{m} \backslash \bar{\Omega}$ such that every planar circular semi arc joining $\bar{x}$ and $b$ intersects $\bar{\Omega}$ in a connected set. (Here we should include the case $b=\infty$ to include the possibility that $\bar{\Omega}$ is already weakly star-shaped and allow the limiting arc of the straight line through 
$\bar{x}$ and $b$. Equivalently we could assume every planar circular arc joining $b$ and $\bar{x}_{0}$ intersects $\bar{\Omega}$ in a connected set (since these are one dimensional sets). These arcs are all arcs with centres on the hyperplane of points equidistant from $\bar{x}$ and $b$. Of course the problem is in the correct choice of $\bar{x}$ and $b$. Each $x \in R^{n} \backslash\{\bar{x}, b\}$ lies on a unique circular arc. Let $v_{\bar{x}, b}(x)$ be the unit tangent vector to this arc at $x$ determined by differentiating at $x$ in the direction moving from $\bar{x}$ to $b$. Then by the proposition and the remark immediately after it, we see that a bounded $C^{1}$ domain $\Omega$ is conformally equivalent to a bounded weakly star shaped domain if and only if there exists $\bar{x} \in \bar{\Omega}$ and $b \in R^{n} \backslash \bar{\Omega}$ such that $\left\langle v_{\bar{x}, b}(x), n(x)\right\rangle \geqslant 0$ on $\partial \Omega \backslash\{\bar{x}\}$. (Note that a conformal transformation preserves angles). From the above we have a quite convenient geometric and analytic characterisation of which bounded $C^{1}$ domains are conformally equivalent to bounded weakly star-shaped domains. We shall also refer to $\bar{x}$ as a centre of $\Omega$.

As a simple consequence of the analytic characterisation and a simple compactness argument, if the $\Omega_{n}$ are bounded domains which are conformally equivalent to bounded weakly star-shaped domains and if $\Omega_{n} \longrightarrow \Omega$ in the $C^{1}$ sense, then $\Omega$ is also conformally equivalent to a bounded weakly star shaped domain unless the points at infinity $b_{n}$ for $\Omega_{n}$ approach $\Omega$ as $n$ tends to infinity. (I do not mean to imply points at infinity are unique.) Note that if $\left\|b_{n}\right\| \longrightarrow \infty$ as $n \longrightarrow \infty$, then $\Omega$ will be weakly star-shaped.

Finally note that, if $\Omega$ is bounded and open and conformally equivalent to a weakly star-shaped domain then $\bar{\Omega}$ is clearly contractible. On the other hand not every contractible domain is conformal to a bounded weakly star shaped domain. (For example, this follows since there are contractible domains for which (1) has a positive solution. We shall meet further examples later.)

\section{NEARLY WEAKLY STAR SHAPED DOMAINS}

In this section, we prove two results. We firstly show that in domains which are almost weakly starshaped, (1) has no positive solution. We also use this and the results of Section 1 to show that there exist bounded domains other than those conformal to bounded weakly star shaped domains for which (1) has no positive solution. This answers a natural question. Note that there are contractible domains for which (1) has a positive solution. (See $[\mathbf{1}, \mathbf{6}]$ or $[\mathbf{1 0}]$.)

THEOREM 1 . Assume that $\Omega$ is a bounded $C^{2}$ weakly star shaped domain and assume $\Omega_{n} \longrightarrow \Omega$ in the $C^{2}$ norm as $n \longrightarrow \infty$. Then (1) on $\Omega_{n}$ has no positive solution for large $n$.

REMARK. The proof is complicated because we do not have a priori bounds for positive solutions.

Proof: By the Pohozaev identity, we see that, if $u_{n}$ is a positive solution of (1) 
on $\Omega_{n}$,

$$
\int_{\partial \Omega}\left\langle x-x_{0}, \nu^{n}(x)\right\rangle\left(\frac{\partial u_{n}}{\partial \nu^{n}}\right)^{2}=0 .
$$

Here it is convenient to change notation slightly and use $\nu^{n}(x)$ for the outward unit normal to a $\Omega_{n}$ at $x$. Suppose we can prove there is a $K>0$ such that

$$
\sup _{\partial \Omega_{n}}\left|\frac{\partial u_{n}}{\partial \nu^{n}}\right| \leqslant K \inf _{\partial \Omega_{n}}\left|\frac{\partial u_{n}}{\partial \nu^{n}}\right|
$$

where $K$ is independent of $n$. Then

$$
\int_{T_{n}}\left\langle\left(x-x_{0}\right), \nu^{n}(x)\right\rangle\left|\frac{\partial u}{\partial \nu^{n}}\right|^{2} \geqslant \mu_{n} H_{n-1}\left(\partial \Omega_{n}\right) K^{2} \inf _{\partial \Omega_{n}}\left|\frac{\partial u_{n}}{\partial \nu^{n}}\right|^{2}
$$

where $T_{n}=\left\{x \in \partial \Omega_{n}:\left\langle\left(x-x_{0}\right), \nu^{n}(x)\right\rangle<0\right\}, \mu_{n}=\inf \left\{\left\langle\left(x-x_{0}\right), \nu^{n}(x)\right\rangle: x \in T_{n}\right\}$ and $H_{n-1}$ is $(n-1)$-dimensional Hausdorff measure. When $x$ is at maximal distance on $\partial \Omega_{n}$ from $x_{0},\left\langle\left(x-x_{0}\right), \nu^{n}(x)\right\rangle=\left\|x-x_{0}\right\|$ and hence we easily see that there is a subset $W_{n}$ of $\partial \Omega_{n} \backslash T_{n}$ with $(n-1)$-dimensional Hausdorff measure bounded below by $\gamma>0$ independent of $n$ and $\left\langle\left(x-x_{0}\right), \nu^{n}(x)\right\rangle \geqslant \ell>0$ independent of $n$ on $W_{n}$. Hence

$$
\int_{\partial \Omega_{n}-T_{n}}\left\langle x-x_{0}, \nu^{n}(x)\right\rangle\left|\frac{\partial u_{n}}{\partial \nu^{n}}\right|^{2} \geqslant \gamma \ell \inf _{\partial \Omega_{n}}\left|\frac{\partial u_{n}}{\partial \nu^{n}}\right|^{2}
$$

Since $\mu_{n} \longrightarrow 0$ as $n \longrightarrow \infty,(4)$ and (5) contradict (2) for $n$ large. Hence it suffices to prove (3).

We first prove (3) assuming that there exist $\delta, K>0$ and independent of $n$ such that

$$
u_{n}(x) \leqslant K \quad \text { if } \quad d\left(x, \partial \Omega_{n}\right) \leqslant \delta .
$$

We prove (6) a little later. To deduce (3), choose a smooth surface $S$ in $\Omega$ close to $\partial \Omega$ (within $\delta / 2$ of $S$ ) such that $S$ intersects each normal to $\partial \Omega$ and this intersection is unique. Let $\widetilde{S}$ be a small closed neighbourhood of $S$ with smooth boundary. Now the $u_{n}$ are uniformly bounded on $\widetilde{S}$ (also uniformly in $n$ ) and hence $-\Delta u_{n}=a_{n} u_{n}$ where $a_{n}$ is uniformly bounded on $\widetilde{S}$ (and uniform in $n$ ). By applying the Harnack inequality (see [9, Corollary 8.21]) on $\widetilde{S}$, we see that on a slightly smaller neighbourhood $V$, there exists $K_{1}$ independent of $n$ such that $\sup _{V} u_{n} \leqslant K_{1} \inf _{S} u_{n}$. We can now apply $W^{2, p}$ estimates (or the $C^{1, \alpha}$ estimates) in [9] to obtain a $C^{1, \alpha}$ independent of $n$ for 
$B_{n}^{-1} u_{n}$ on $W_{n}$, where $W_{n}$ is the region bounded by $S$ and $\partial \Omega_{n}$ and $B_{n}=\sup _{W_{n}} u_{n}$. There are a couple of points to be noted here. Firstly we need to follow the discussion in $[9$, p.237-230 (see also p.98 there) ] to check the bounds can be made independent of $n$. The argument is a localisation argument. Secondly, to obtain the bounds near $S$ we use that our earlier Harnack inequality estimate implies $B_{n}^{-1} u_{n}$ is bounded on $W_{n} \cup V$ and hence we can use interior estimates near $S$. Moreover it follows that we have a positive lower bound for $-\left(1 / B_{n}\right) \frac{\partial u_{n}}{\partial \nu^{n}}$ on $\partial \Omega_{n}$ where, as before, $\nu^{n}$ is the outward unit normal. To see that this is true, suppose by way of contradiction that it is false. Then there exists a subsequence of the $B_{n}^{-1} u_{n}$ converging uniformly in the $C^{1}$ norm to a solution $u$ of $\Delta u=\widehat{a} u$ on the region $W$ between $S$ and $\partial \Omega$ such that $u$ is bounded by 1 , has a positive lower bound on $S$ and has a zero normal derivative at some point of $\partial \Omega$ (and $u=0$ on $\partial \Omega$ ). Here $\widehat{a}$ is bounded. There are two points here. Firstly, we extend the $u_{n}$ to be zero outside $\Omega_{n}$ to obtain functions on a fixed domain. Secondly, if $B_{n}^{-1} u_{n}$ converge uniformly to zero on $S$, then, by the Harnack inequality, $B_{n}^{-1} u_{n}$ converge uniformly to zero on $V$ and hence $u$ vanishes identically on $V$. By the Harnack inequality, it follows that $u=0$ on $W$. This is impossible since $B_{n}^{-1} u_{n}$ converges uniformly and $\sup _{W_{n}} B_{n}^{-1} u_{n}=1$. However by the Hopf maximum principle applied to $u$ on $W, \frac{\partial u}{\partial \nu}<0$ on $\partial \Omega$. Hence we have a contradiction. This proves that $-B_{n}^{-1} \frac{\partial u_{n}}{\partial \nu^{n}}$ has a positive lower bound on $\partial \Omega_{n}$ (uniformly in $n$ ). Since we also have a bound for $\left|B_{n}^{-1} \frac{\partial u_{n}}{\partial \nu^{n}}\right|$ on $\partial \Omega_{n}$ (uniform in $n$ ), this proves (3).

It remains to prove (6). This is a more geometric argument. By our convergence assumptions there is a $\mu>0$ independent of $n$ and $x_{1}$ such that each point $x_{1} \in \partial \Omega_{n}$ is on the boundary of a ball $B_{x_{1}, n}$ in $R^{m} \backslash \Omega_{n}$ of radius $\mu$ (that is a touching ball). Here $\mu$ is rather small. We use an inversion in $B_{x_{1}, n}$. Under this inversion, $\Omega_{n}$ becomes a $C^{2}$ domain $\widetilde{\Omega}_{x_{1}, n}$ contained in $B_{x_{1}, n}$ and touching $\partial B_{x_{1}, n}$ at $x_{1}$. Moreover if $x_{1}^{n} \in \partial \Omega_{n}$ converges to $x_{1} \in \partial \Omega$ as $n \longrightarrow \infty, \widetilde{\Omega}_{x_{1}, n}^{n}$ converges in the $C^{2}$ norm to $\widetilde{\Omega}_{x_{1}}$ as $n \longrightarrow \infty$ uniformly in $x_{1}$. ( $\widetilde{\Omega}_{x_{1}}$ is the image of $\Omega$ under the inversion in the ball $\left.B_{x_{1, n}} \in R^{m} \backslash \Omega\right)$. Now by the conformal invariance, $v_{n}(y) \equiv\left(\left\|y-\widehat{x}_{0}^{n}\right\|\right)^{2-m} u_{n}\left(\left(y-\widehat{x}_{0}^{n}\right) /\left\|y-\widehat{x}_{0}^{n}\right\|^{2}\right)$ is a positive solution of (1) on $\widetilde{\Omega}_{x_{1}, n}$ with $v_{n}(y)=0$ on $\partial \widetilde{\Omega}_{x_{1}, n}$. (Here $\widehat{x}_{0}^{n}$ is the centre of $B_{x_{1}, n}$.) We can use the method of sliding planes (see as in [8]) to $v_{n}$ on $\widetilde{\Omega}_{x_{1}, n}$ and obtain that $v_{n}$ increases in certain directions. By repeating the argument of $\mathrm{De}$ Figueredo, Nussbaum and Lions [5, p.51-53], we see that there exists $\varepsilon, \gamma, C>0$ independent of $n$ such that if $x \in \Omega_{\varepsilon, n}=\left\{x \in \bar{\Omega}_{n}: d\left(x, \partial \Omega_{n}\right)<\varepsilon\right\}$ there exists a measurable set $I_{x, n}$ with $\mu\left(I_{x, n}\right) \geqslant \gamma$ (where $\mu$ denotes Lebésque measure) such that $I_{x, n} \subseteq \Omega_{n} \backslash \Omega_{1 / 2 \epsilon, n} \equiv T_{n}$ and $u_{n}(\xi) \leqslant C u_{n}(S)$ if $\xi \in I_{x, n}$. (That $\widetilde{\Omega}_{x_{1}^{n}, n}$ converges in 
$C^{2}$ to $\widetilde{\Omega}_{x_{1}}$ ensures that we can do this uniformly in $n$ ). Hence we see that

$$
\int_{T_{n}} u_{n}^{p} \geqslant \gamma C^{-p} u_{n}(x)^{p} \quad \text { if } \quad x \in \Omega_{\varepsilon, n} .
$$

On the other hand, by scalar multiplying (1) (for $\dot{\Omega}=\Omega_{n}$ ) by $\phi_{n}$, the positive eigenfunction of $-\Delta$ on $\Omega_{n}$ (for Dirichlet boundary conditions on $\partial \Omega_{n}$ ), normalised so that $\left\|\phi_{n}\right\|_{2, \Omega_{n}}=1$ we see that $\int_{\Omega_{n}}\left(u_{n}^{p}-\lambda_{1}\left(\Omega_{n}\right) u_{n}\right) \phi_{n}=0$ where $\lambda_{1}\left(\Omega_{n}\right)$ is the eigenvalue corresponding to $\phi_{n}$. Since $\lambda_{1}\left(\Omega_{n}\right) \longrightarrow \lambda_{1}(\Omega)$ as $n \longrightarrow \infty$ (by the theory of domain variation as in [3] or [11]) and since $y^{p}-\gamma y$ is bounded below on $[0, \infty)$ whenever $\gamma>0$, it follows easily that $\int_{\Omega_{n}} u_{n}^{p} \phi_{n} \leqslant K$ where $K$ is independent of $n$. Suppose that $M$ is a compact subset of $\Omega$. By domain variation again, $\phi_{n} \longrightarrow \phi$ uniformly on $M$ where $\phi$ is the first eigenfunction of $-\Delta$ on $\Omega$. Hence there is a $K_{1}>0$ such that $\phi_{n}(x) \geqslant K_{1}$ if $x \in M$ and $n$ is large. Hence

$$
\int_{M} u_{n}^{p} \leqslant K_{2}
$$

where $K_{2}$ is independent of $n$. If we choose $M=\left\{x \in \Omega: d\left(x_{1} \partial \Omega\right) \geqslant \varepsilon / 4\right\}$ then $T_{n} \subseteq M$ for large $n$ and hence the result follows from (7) and (8). This completes the proof.

REmarks 1. We do not know if the result is true for changing sign solutions. Note that, by the Pohojaev identity argument, there are no changing sign solutions if $\Omega$ is weakly star shaped.

It follows that if $\Omega_{n} \longrightarrow \Omega$ in the $C^{2}$ sense and $\Omega$ is conformally equivalent to a bounded $C^{2}$ weakly-star-shaped domain, then (1) has no positive solution on $\Omega_{n}$ for large $n$. This follows because, if $\widetilde{\Omega}$ is conformally equivalent to $\Omega$ where both are bounded domains, then (1) has a positive solution on $\Omega$ if and only if it has a positive solution on $\widetilde{\Omega}$. This is obvious for translations, rotations and rescalings so that we need only consider inversions in a ball of radius 1 centre zero. Now if $\widetilde{\Omega}$ is the image of $\Omega$ under this inversion, it is well known and easy to see that $u$ is a solution on $\Omega$ if and only if $v(y)=\|y\|^{2-m} u\left(y /\|y\|^{2}\right)$ is a positive solution on $\left\{x /\|x\|^{2}: x \in \Omega\right\}$ and hence our claim follows.

It is a natural question to ask whether being conformal to a weakly star shaped set characterises the domains $\Omega$ for which (1) has no positive solution. We now construct an example to show that this is not the case.

To construct such an example we shall construct a smooth family of domains $W_{t}, t \in[0,1]$ such that $W_{0}$ is weakly star shaped and $W_{1}$ is not conformal to a bounded weakly star shaped domain. If we can construct such a family with $T=\left\{t \in[0,1]: W_{t}\right.$ 
is conformally equivalent to a bounded weakly star shaped domain $\}$ is closed, then we shall have the required example. This follows because if $\gamma=\sup T$, then $\gamma<1, W_{\gamma+\varepsilon}$ is not conformally equivalent to a bounded weakly star shaped domain while by one of our remarks after Theorem 1 , the critical exponent problem on $W_{\gamma+\varepsilon}$ has no positive solution as required (because $W_{\gamma+\varepsilon}$ is a $C^{2}$ small perturbation of $W_{\gamma}$ ).

We now consider when $T$ is closed. If $W_{t_{n}}$ is conformal to a bounded weakly star shaped domain for all $n$, and $t_{n} \longrightarrow t$ as $n \longrightarrow \infty$, then by the remarks at the end of Section 1, the only way that $W_{t}$ can fail to be conformal to a bounded weakly star shaped domain is that the point at infinity $b_{n}$ of $W_{t_{n}}$ satisfies $b_{n} \longrightarrow \partial W_{t}$ as $n \longrightarrow \infty$. We show that this does not occur if we choose the family $W_{t}$ carefully. This will complete the construction of our example.

We construct $W_{0}$ as follows. Let $T_{k}^{\prime}, k=1, \cdots, s$ (where $s \geqslant 4$ ) be open balls of small radius with centres on the ball of radius 4 and so that the centres are well spaced. We define $W_{0}=B_{1} \cup \bigcup_{k=1}^{s} \overline{c o}\left(T_{k} \cup\{0\}\right)$. It is easy to see that $W_{0}$ is weakly star shaped but the only point it is weakly star shaped from is zero. We can easily smooth the corners without affecting those properties. We note that $W_{0}$ can not be conformal to a weakly star shaped domain with the "point at infinity" $b$ close to $\partial W_{0}$. To see this, note that, whatever the location of the centre $a$, there must be at least $s-1$ circular arcs, each in one of the $\overline{c o}\left(T_{k} \cup\{0\}\right)^{\prime} s$ joining $\|x\|=2$ to $\|x\|=3$. However, if the $T_{k}^{\prime} s$ are chosen small, these circular arc's must have small curvature. However, some elementary geometry shows that the only circular arc joining $a$ and $b$ having small curvature between $a$ and $b$ must have direction nearly parallel to $b-a$ in the set $\{x:\|x\| \leqslant 4\}$. Here we use that $\|b\|$ is not large. Provided the centres of the $T_{k}^{\prime} s$ are well spaced, this clearly leads to contradiction. Thus the point $b$ "at infinity" can not be near $\partial W_{0}$. For future reference note that this argument shows that the point $b$ "at infinity" must be large, the circular arcs must have small curvature within our set (except possibly for those in the $T_{k}$ containing the centre $a$ ) and the centre $a$ which is the intersection points of all those arcs with small curvature must be near zero.

We now define the $W_{t}$ for $t>0$ by squeezing each of the tubes $\overline{c o}\left(\{0\} \cup T_{k}\right)$; or more precisely the part of the tubes with $1<\|x\|<2$. We explain this by drawing a two dimensional cross section of a tube. (We do the squeezing in a symmetric way within a tube and we squeeze each tube in the same way). We could easily give a formula for the squeezing but the diagram is more informative.

In the diagrams, we have not drawn them to scale (by widening the tube) to make them easier to understand. Since the $W_{t}^{\prime} s$ are the same as $W_{0}$ for $2 \leqslant\|x\| \leqslant 3$, the argument in the previous paragraph shows that, if $W_{t}$ is conformal to a bounded weakly star shaped domain, then the point at infinity $b_{t}$ is not near the boundary of $W_{t}$ and this holds uniformly in $t$. (Remember that the part of $W_{t}$ in $\{x: 2<\|x\|<3\}$ is 

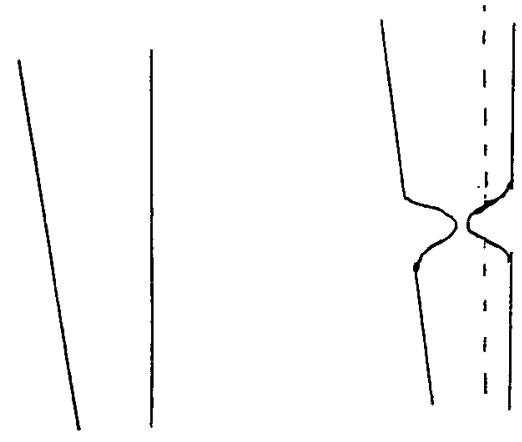

Figure 1

independent of $t$.) Hence, as we saw earlier, $\left\{t: W_{t}\right.$ is conformal to a weakly star shaped bounded domain $\}$ is closed and hence we are finished if we prove that $W_{t}$ becomes not conformal to a bounded star shaped domain before the two sides of $\overline{c o}\left(T_{k} \cup\{0\}\right)$ are squeezed together. To see this, note that, by the remarks at the end of the previous paragraph, the point at infinity $b_{t}$ must be large, $a_{t}$ must be small and the circular arcs must be nearly straight within $W_{t}$. Now consider $x$ in $W_{0}$ with $2<\|x\|<3$ and $x$ very close to the boundary. Since the circular arcs are very straight, $a_{t}$ is small and $b_{t}$ is large, it is easy to see that the semi-arc joining $a_{t}$ to $b_{t}$ through $x$ will cross $\partial W_{t}$ at three points when the squeezing is significant (one with $\|x\|$ close to 4 and two where the circular arc crosses the region being squeezed (as in the diagram). Hence the connectedness condition fails and $W_{t}$ is not conformal to a weakly star shaped bounded domain. This completes the construction of the counterexample.

Finally, another example is mentioned in Section 3, while a higher dimensional version of the example in [4] could almost certainly be used to obtain another example (though there would be a good deal of tedious geometric arguments to justify them). The example in [4] (or more precisely a higher dimensional analogue) probably also provides an example where we make a small perturbation of a star-shaped domain and obtain domains not conformal to bounded weakly star shaped domains.

\section{An example}

Here we show that our geometric techniques can be used to give a simple proof of a result much stronger than one in Rodriguez, Comte and Lewandowski [12]. In fact, we answer two open questions in [12].

Let $B$ be the open ball in $R^{m}, \varepsilon>0, \rho<-1, \ell \in(0,1)$. We define

$$
\begin{aligned}
& C_{\varepsilon}=\left\{\left(x^{\prime}, x_{m}\right): x^{\prime} \in R^{m-1},\left\|x^{\prime}\right\| \leqslant \varepsilon\left(x_{m}-\rho\right)(1-\rho)^{-1}\right\} \\
& \Omega_{\varepsilon}=B \backslash\left\{x \in C_{\varepsilon}: x_{m} \geqslant \ell\right\} .
\end{aligned}
$$


THEOREM 2. For $\Omega=\Omega_{\varepsilon}$, (1) has no solution.

REMARK. If we replace $u^{p}$ by $|u|^{p-1} u$, the result also applies to sign changing solutions.

Proof: Except for a technical smoothness problem, it suffices to show that $\Omega_{\varepsilon}$ is conformal to a bounded weakly star-shaped domain. (Note that this contradicts a comment in $[12, \mathrm{p} .245])$. We choose $a=(0,-B)$ where $B \in(0,1)$ and is close to 1 and $b=(0, \alpha)$ where $\alpha$ is larger than 1 . Here $a$ is the centre and $b$ is the point at infinity. We shall specify $\alpha$ more closely later. Note that, unlike [12], we do not require $\varepsilon$ to be small. It suffices to show that every planar circular semi arc joining $a$ and $b$ intersects $\Omega_{\varepsilon}$ in a connected set. By the symmetry of $\Omega_{\varepsilon}$ under rotations about the $e_{m}$ axis, we see that this reduces to a two dimensional problem. Hence we can set $m=2$. It is now elementary geometry. Any circular semi arc joining $a$ and $b$ is part of a circle with centre $(s,(\alpha-B) / 2)$, containing $a$ and $b$. This circle will intersect the unit circle $T$ $x_{1}^{2}+x_{2}^{2}=1$ in exactly two points, once on each of the two semi arcs joining $a$ and $b$. (It must intersect more than once because $b$ is outside $T$ while $a$ is inside $T$ ). We need only consider semi arcs in $x_{1} \geqslant 0$. Suppose we can prove the circular semi arc $P$ through the corner where a straight edge meets a curved edge does not intersect any straight edge (or other corner). If we prove this, a simple connectedness argument implies that semi arcs to the "right" of $P$ will not intersect the straight edge of $\partial \Omega_{\varepsilon}$ and will intersect the curve edge exactly once while semi arcs to the "left" of $P$ will not intersect the curved part of $\partial \Omega_{\varepsilon}$. Now our semi arcs to the "right" of $P$ will be of the form $x_{1}=g\left(x_{2}\right)$ for $\alpha \geqslant x_{2} \geqslant-B$ where $g$ decreases for $\alpha \geqslant x_{2} \geqslant(\alpha-B) / 2$ and $g$ is even about $x_{2}=(\alpha-B) / 2$. On the other hand the boundary of $C_{\varepsilon}$ in $x_{1}>0$ is an increasing function of $x_{2}$ for $x_{2}>\ell$. Hence if $(\alpha-B) / 2 \leqslant \ell$, we see that the semi arc can meet the part of $C_{\varepsilon}$ in $x_{1}>0$ in at most one point (and $P$ cannot meet again this part of $C_{\varepsilon}$ ). The claim on our semi arcs now follows easily provided $(\alpha-B) / 2 \leqslant \ell$ which is true if $\alpha$ and $B$ are chosen suitably (for $\ell \in(0,1)$ ).

There is one technical point in our proof. $\Omega_{\varepsilon}$ will be conformal to a bounded weakly star shaped domain $\widetilde{\Omega}_{\varepsilon}$ with corners but still with the rotational symmetry. To prove non-existence, we need to check that $\widetilde{\Omega}_{\varepsilon}$ is smooth enough so that the Pohozaev identity holds. This follows by the argument in [3, p.655-657]. This completes the proof.

\section{REMARKS.}

1. We could also take $a=(-1,0)$ and $b=(1,0)$ where the geometry is a little simpler. However, our choice of $a$ and $b$ has the advantage we can use them for many smooth perturbations of $\Omega_{\varepsilon}$.

2. With a little care, it can be shown that $\Omega_{\varepsilon}$ is still conformal to a bounded 
weakly star shaped domain if $\ell=1$ or indeed $\ell$ is slightly larger than 1 . However it can be shown that $\ell \leqslant 1$ is the best condition on $\ell$ we can allow for $\Omega_{\varepsilon}$ to be conformal to a weakly star shaped domain if we want a condition independent of $\varepsilon$ and $\ell$. Note also that we could replace $C_{\varepsilon}$ by $\left\{\left(x^{\prime}, x_{m}\right):\left\|x^{\prime}\right\| \leqslant \mu\right\}$ and the arguments are still valid (though here we can not go above $\ell=1$ ). If $\ell<1$, we could even allow a very small "knob" at the end of the tube. In this case, the domains are quite close to those in Dancer [1] or Ding [6] and it shows the importance of the "knob" at the end of the tube in the examples there. Note that the methods in [1] can be modified to cover cases where the spherical "knob" does not lie at the centre of the outer ball. These examples seem to suggest it is very difficult to decide for which $\Omega(1)$ has a positive solution.

3. It is possible, though a lot of tedious geometrical arguments are needed, to show that, if we round off the corners in our previous example, then as we increase $\ell$ we obtain a different example where (1) has no solution even though $\Omega$ is not conformal to a bounded weakly star-shaped domain. (The difficulty is in establishing that the obvious choices of the centre and the point at infinity are optimal.)

4. In general, conformal equivalences seem to be very useful for domains with one spike or reentrant region but less useful for domains with several spikes (or several reentrant regions).

\section{REFERENCES}

[1] E.N. Dancer, 'A note on an equation with critical exponent', Bull. London Math. Soc. 20 (1988), 600-602.

[2] E.N. Dancer, 'The effect of domain shape on the number of positive solutions of certain nonlinear equations', J. Differential Equations. 74 (1988), 120-156.

[3] E.N. Dancer, 'On the influence of domain shape on the existence of large solutions of some superlinear problems', Math. Ann. 285 (1989), 647-669.

[4] E.N. Dancer and K. Zhang, 'Uniqueness of solutions for some elliptic equations and systems in nearly star shaped domains', (preprint).

[5] D. De Figuerdo, P.L. Lions and R. Nussbaum, 'A priori estimates and existence of positive solutions of semilinear elliptic equations', J. Math. Pure Appl. 61 (1982), 41-63.

[6] W. Ding, 'Positive solutions of $-\Delta u=u^{(N+2) /(N-2)}$ on contractible domains', J. Partial Differential Equations 2 (1989), 83-88.

[7] E. Dold, Algebraic topology (Springer Verlag, Berlin, Heidelberg, New York, 1970).

[8] B. Gidas, W.M. Ni and L. Nirenberg, 'Symmetry and related properties by the maximum principle', Comm. Math. Phys. 68 (1979), 209-213.

[9] D. Gilbarg and N. Trudinger, Elliptic equations of second order (Springer Verlag, Berlin, Heidelberg, New York, 1983).

[10] D. Passaseo, 'Multiplicity of positive solutions of nonlinear elliptic equations with critical Sobolev exponent on some contractible domains', Manuscripta Math. 65 (1989), 147-166. 
[11] J. Rauch and M. Taylor, 'Potential and scattering theory in wildly perturbed domains', J. Funct. Anal. 18 (1975), 27-59.

[12] A. Carpio Rodriguez, M. Comte and R. Lewandowski, 'A non-existence result for a nonlinear equation involving critical Sobolev exponent', Analyse Nonlinéaire 9 (1992), 243-261.

[13] J. Vaisala, Lectures on n-dimensional quasi-conformal mappings, Lectures notes in mathematics 229 (Springer Verlag, Berlin, Heidelberg, New York, 1971).

School of Mathematics and Statistics

University of Sydney

New South Wales 2006

Australia 